\title{
Transient Alkaline Hyperphosphatasaemia in an Adult: Biochemical Peculiarities
}

\author{
Christian M. Schambeck. Andrea Kopp, Gertrud Mora-Maza and Franz Keller
}

Zentrallabor der Medizinischen Klinik, Bayerische Julius-Maximilians-Universität Würzburg, Würzburg, Germany

Summary: We report on a 27-year-old healthy female with transient hyperphosphatasaemia of adulthood (it is the eighth case ever recorded). A maximum alkaline phosphatase activity of $1950 \mathrm{U} / 1,11$-fold the upper reference limit, was measured. The activity normalized within 11 weeks. Electrophoresis revealed the typical pattern for alkaline phosphatase isoenzymes observed in transient hyperphosphatasaemia of infancy: a fast-migrating liver isoenzyme and a bone isoenzyme. Contrary to the findings in transient hyperphosphatasaemia of infancy the liver isoenzyme did not precipitate with wheat-germ lectin whereas the bone isoenzyme partially bound to lectin.

Biochemical features of transient hyperphosphatasaemia in an adult may be different from those in infancy. Recognition of an atypical pattern could help avoid unnecessary extensive investigations.

\section{Introduction}

Since the first report published by Assanti et al. (1) there have been many case reports and a few longer series dealing with a marked, but transient, increase of alkaline phosphatase in children $(2,3)$. Transient hyperphosphatasaemia in infancy is characterized by a typical pattern of alkaline phosphatase isoenzymes: a liver isoenzyme shows an enhanced electrophoretic mobility and increased binding to wheat-germ lectin by affinity electrophoresis. The bone isoenzyme, however, does not show this remarkable increase in mobility. There is no evidence of liver and bone disease. The etiology of transient hyperphosphatasaemia remains an enigma.

This syndrome has been also recorded in adults but it seems a very rare event. Only seven cases have been published to our knowledge (4-10). A more precise biochemical characterization of the atypical alkaline phosphatase isoenzymes was performed in just two cases $(4,9)$. Here we report on an eighth adult whose alkaline phosphatase isoenzymes revealed some biochemical differences compared to transient hyperphosphatasaemia in infancy.

\section{Materials and Methods}

\section{Patients}

We analyzed sera not only from the adult whose hyperphosphatasaemia we investigated, but also from infants. These infants were two boys and two girls, ages nine to 29 months (median 20 months), whose increase in serum alkaline phosphatase had been noted as an incidental and unexpected finding during the course of hospital admission for a disorder not normally associated with such an increase. In order to compare the electrophoretic mobility of the bone isoenzyme in our adult with that in different bone diseases, we used sera from 3 patients with hyperparathyroidism, 3 patients with malignant disease and 1 patient with connective tissue disorder. We used pooled sera from patients with cholestasis as control serum. Electrophoresis indicated two well-defined bands, the soluble liver alkaline phosphatase isoenzyme (the liver isoenzyme circulating as a soluble homodimer) and the membrane-bound liver alkaline phosphatase isoenzyme (the liver isoenzyme anchored to fragments of the liver cell membrane, often called "high molecular mass liver isoenzyme", "fast-liver isoenzyme", "bile isoenzyme") (10).

Measurement of total alkaline phosphatase and electrophoresis of alkaline phosphatase isoenzymes

Measurement of total alkaline phosphatase activity was performed on the automatic chemical analyzer Hitachi 747 (Boehringer Mannheim, Germany) using $p$-nitrophenyl phosphate as substrate at $25^{\circ} \mathrm{C}$ (11). Electrophoresis was carried out with SEBIA agarose gels (SEBIA, Issy-les-Mouligneux, France): the $0.9 \%$ agarose gels were used with and without $0.05 \mathrm{~g} / 1$ of wheatgerm lectin. The substrates were indolyl phosphate, $\mathrm{MgCl}_{2}$ and nitro blue tetrazolium chloride monohydrate in ethanolamine buffer ( $\mathrm{pH}$ 9.8). The gels were used with a Tris-barbital buffer $(5.75 \mathrm{~g} / 1$ Tris, $1.47 \mathrm{~g} / 1$ barbital, $8.24 \mathrm{~g} / 1$ sodium barbital and $0.012 \mathrm{~g} / 1$ sodium azide, $\mathrm{pH} 9.1$ ). The gels were scanned at 600 $\mathrm{nm}$ with an Appraise densitometer (Beckman, Brea, USA). The area under the curve and the corresponding isoenzyme activities were computed by the densitometer.

The within- and between-gel reproducibilities of the scanned areas under the curve were checked by means of serum pool controls (total alkaline phosphatase activity 400-800 U/1, isoenzyme activities ranged from $7 \%$ to $90 \%$ of total alkaline phosphatase activity). The within-gel variation of the gels with and without wheat-germ lectin $(n=3)$ ranged from $1 \%$ to $11 \%$ and $1 \%$ to $9 \%$, respectively. The between-gel variation $(\mathrm{n}=3)$ did not exceed $9 \%$.

Biochemical characterization of alkaline phosphatase isoenzymes

For treating the samples with neuraminidase, $20 \mu \mathrm{l}$ of a neuraminidase solution from Clostridium perfringens (Beckman, Brea, USA) were added to $100 \mu \mathrm{l}$ of serum and incubated for 30 minutes at $37^{\circ} \mathrm{C}$. Incubation with polyclonal anti-placental antiserum (Beckman, Brea, USA) was performed by adding $20 \mu \mathrm{l}$ 
of the antibody solution to $100 \mu \mathrm{l}$ of serum. To resolve proteinbound isoenzymes as well as polymers of liver isoenzymes of alkaline phosphatase, $5 \mu \mathrm{l}$ ficin from fig tree latex (Beckman, Brea, USA) were added to $100 \mu \mathrm{l}$ of serum and incubated for 30 minutes at $37^{\circ} \mathrm{C}$. Serum was treated with $\alpha$ - $L$-fucosidase from bovine kidney: $10 \mu \mathrm{l}$ from a fucosidase solution (5-15 units per mg protein at $\mathrm{pH} 5.5$ at $25^{\circ} \mathrm{C}$, Sigma Chemical Co, St. Louis, USA) were added to $90 \mu \mathrm{l}$ serum. Then, the mixture was incubated for 24 hours at $37^{\circ} \mathrm{C}$. Inhibition by $L$-phenylalanine, $L$-homoarginine and $L$-leucine (Sigma, St. Louis, USA) was determined by addition of these amino acids to the assay mixture to a final concentration of $5 \mathrm{mmol} / \mathrm{l}$. The percentage inhibition was calculated with reference to the activity obtained in the absence of these amino acids. Heat-stability of alkaline phosphatase was determined as the half-inactivation time of the enzyme on incubation of $56^{\circ} \mathrm{C}$ for various time intervals. After incubation, samples were immediately put in melting ice in order to stop the thermodenaturation. The residual activity was then assayed and the half-inactivation time was determined from a graph of $\log$ (activity) versus time. To identify bone alkaline phosphatase, electrophoreses of samples after heat inactivation at $56^{\circ} \mathrm{C}$ for 10 minutes were examined.

\section{Results}

A 27-year-old female blood donor showed alkaline phosphatase increasing to 8 -fold the upper reference limit (alkaline phosphatase $=1420 \mathrm{U} / \mathrm{l}$ ) on October 13, 1995. Remaining laboratory investigations revealed normal values for electrolytes, alanine and aspartate aminotransferase, $\gamma$-glutamyltransferase, lactate dehydrogenase, bilirubin, creatinine, complete blood count, carcinoembryonic antigen, $\alpha$-foetoprotein. The woman did not complain of any symptoms and the physical examination did not show any abnormalities. Six days later alkaline phosphatase activity of $1950 \mathrm{U} / 1$ was measured,

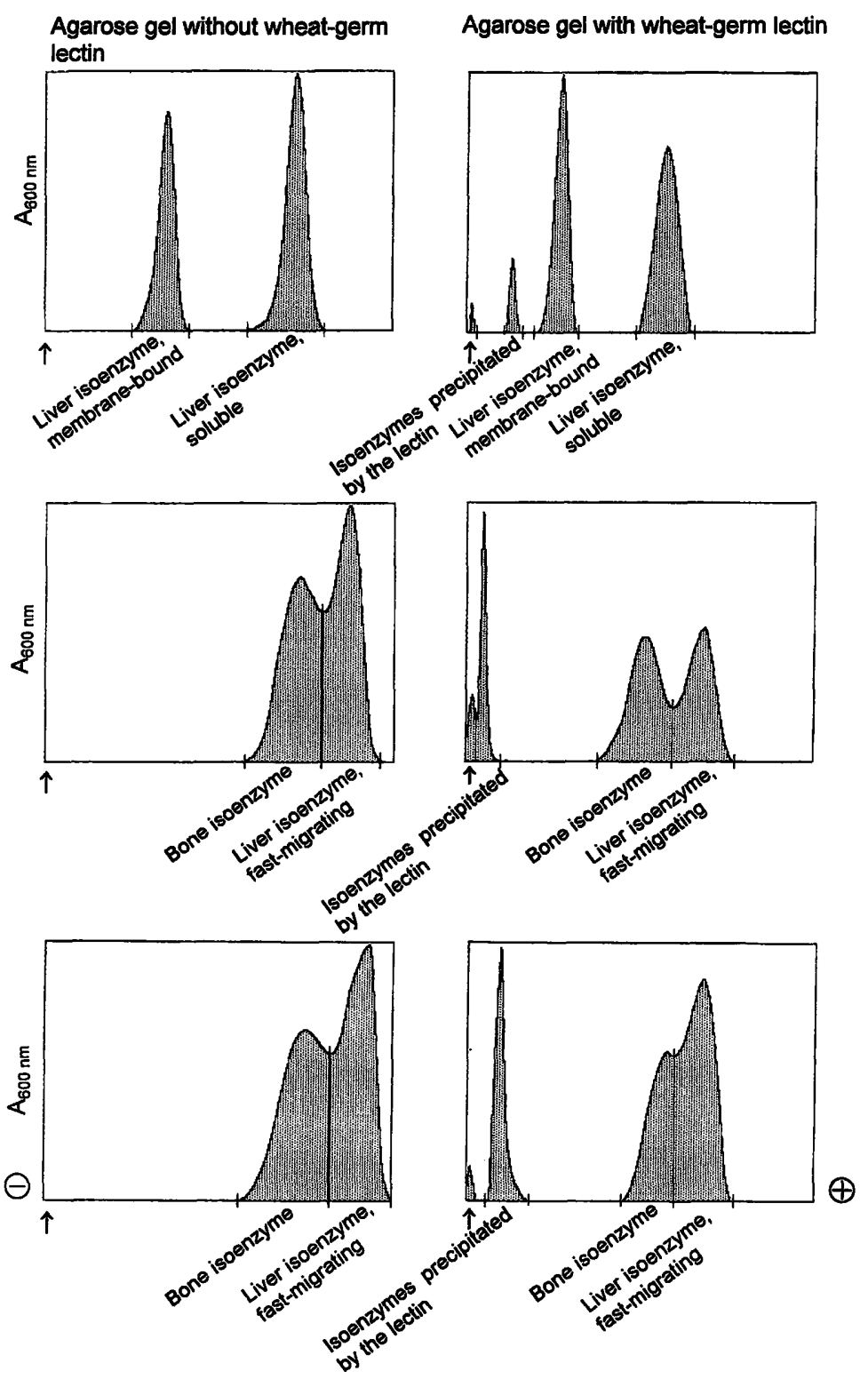

Fig. 1 Densitometric scanning of agarose gels after electrophoretic separation of alkaline phosphatase isoenzymes. The agarose gels were used without (left column) and with (right column) wheat-germ lectin. The samples used were control serum (a), serum from a 1.3-year-old girl with transient hyperphosphatasaemia (b) and serum from the 27-year-old female with transient hyperphosphatasaemia (c). The arrow indicates the origin. 
11-fold the upper reference limit. At the time two bands were detected by electrophoresis of alkaline phosphatase isoenzymes, one sharply defined band with an increased anodal mobility and one band with a more diffuse appearance and mobility of bone-derived alkaline phosphatase (fig. 1). An alkaline phosphatase activity of 303 U/1 was measured on November 14, 1995. The diffuse, but not the fast-migrating, band had nearly disappeared. Blood was also drawn from some relatives, i. e. the 29year-old sister, the 55-year-old mother and the 63-yearold father. Normal electrophoretic patterns of the relatives excluded familial inherited hyperphosphatasaemia.

We decided on an extensive clinical examination if a new blood examination revealed this atypical alkaline phosphatase isoenzyme again. However, alkaline phosphatase activity normalized within 11 weeks (alkaline phosphatase $=87$ U/1 on January 29, 1996) and no biochemical abnormality other than the alkaline phosphatase electrophoretic pattern of a healthy person could be found. We diagnosed transient hyperphosphatasaemia.

The atypical fast-migrating band was characterized by a liver isoenzyme-like half-life of $390 \mathrm{~s}$ at $56^{\circ} \mathrm{C}$. It was not inhibited by $L$-phenylalanine or $L$-leucine ( $3 \%$ and $5 \%$ inhibition, respectively), but partially by $L$-homoarginine (26\% inhibition). This band did not show any binding to wheat-germ lectin-agarose, whereas the more diffuse band identified as a bone isoenzyme was partially affected by wheat-germ lectin-agarose. The fastmigrating liver isoenzyme of the infants bound to wheatgerm lectin to a greater (or an equal) extent than the bone isoenzyme (fig. 1 and tab. 1). The bone isoenzyme showed a slightly reduced mobility compared to the bone isoenzymes in the infants and in the patients with various bone diseases. Neither of the isoenzymes of the adult reacted to polyclonal anti-placental antiserum or ficin. Their electrophoretic mobility was clearly reduced by digestion with neuraminidase.

Tab. 1 Lectin-binding profiles of alkaline phosphatase isoenzymes in patients with transient hyperphosphatasaemia

\begin{tabular}{|c|c|c|c|c|}
\hline \multirow[t]{2}{*}{ Sex } & \multirow[t]{2}{*}{$\begin{array}{l}\text { Age } \\
\text { (a) }\end{array}$} & \multirow{2}{*}{$\begin{array}{l}\text { Alkaline } \\
\text { phosphatase } \\
\text { activity } \\
\text { (U/l) }\end{array}$} & \multicolumn{2}{|c|}{$\begin{array}{l}\text { Non-precipitated fraction of } \\
\text { isoenzyme activity }(\%)^{*}\end{array}$} \\
\hline & & & $\begin{array}{l}\text { Bone } \\
\text { alkaline } \\
\text { phosphatase }\end{array}$ & $\begin{array}{l}\text { Fast- } \\
\text { migrating } \\
\text { liver alkaline } \\
\text { phosphatase }\end{array}$ \\
\hline$\widehat{\delta}$ & 0.8 & 1490 & 59 & $<5$ \\
\hline 오 & 1.3 & 7040 & 85 & 73 \\
\hline 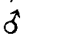 & 2.0 & 1720 & 74 & 73 \\
\hline q & 2.4 & 6050 & 94 & 76 \\
\hline q & 27 & 1950 & 54 & $>95$ \\
\hline
\end{tabular}

* Percentage of isoenzyme activity was derived from the densitometric scan. Then, percentage as determined by affinity electrophoresis was divided by percentage as determined by agarose electrophoresis without wheat-germ lectin.
Treatment with $\alpha$ - $L$-fucosidase decreased very slightly the mobility of the fast-migrating liver isoenzyme in the adult as well as in the infants. However, the soluble liver alkaline phosphatase isoenzyme of our control serum was also slightly retarded.

\section{Discussion}

An elevated serum alkaline phosphatase activity in adults may lead to extensive investigations when common causes of such an increase can be excluded. Thus, malignant disease could be the underlying process of an alkaline phosphatase increase. Transient hyperphosphatasaemia has to be considered, but it is a rare phenomenon. It usually occurs in infants. However, it has also been observed in adults. Electrophoretic separation of alkaline phosphatase isoenzymes may uncover for transient hyperphosphatasaemia an isoenzyme pattern similar to that of transient hyperphosphatasaemia in infancy: a fast-migrating liver isoenzyme with an at least partial binding to wheat-germ lectin (10). Then, the benign nature of transient hyperphosphatasaemia is recognized early and a watch-and-wait policy would be an acceptable procedure. Our findings in an adult with a transient hyperphosphatasaemia imply that biochemical characteristics of the alkaline phosphatase isoenzymes can differ from the pattern in infants.

Treating the samples with neuraminidase, polyclonal anti-placental antiserum and ficin, inhibition by some amino acids, and heat-stability identified the fast-migrating isoenzyme of our adult as a liver alkaline phosphatase isoenzyme. This atypical fast-migrating liver isoenzyme did not bind to wheat-germ lectin, whereas the fast-migrating liver isoenzymes of our infants with a transient hyperphosphatasaemia showed even a more substantial binding by affinity electrophoresis than their bone alkaline phosphatase isoenzymes. This uniform pattern of lectin-binding within infants contrasts with the considerable variation in the non-precipitated fraction of the fast-migrating liver alkaline phosphatase.

Crofton demonstrated both liver and bone alkaline phosphatase isoenzymes in a transient hyperphosphatasaemia of infants to be more readily bound by wheat-germ lectin compared with age-matched controls. Furthermore, there was strong evidence that lectin binding of the alkaline phosphatase liver isoenzyme correlated very well with electrophoretic mobility in infants with a transient hyperphosphatasaemia. This observation corresponds well with the findings in our infants, but obviously contrasts with the situation in our adult. Excessively sialylated isoenzymes might cause the peculiarities in infants. The high sialic acid content of alkaline phosphatase isoenzymes was suggested to provide protection from hepatic clearance (3). Therefore, the high electrophoretic mobility of the fast-migrating liver isoenzyme in our 
case cannot be due to the augmented negative charges of a sialic acid excess. Moreover, $L$-fucose, another terminal sugar residue, did not contribute to the increased mobility as our experiments showed.

Another case of a transient hyperphosphatasaemia in an adult already revealed a situation different from hyperphosphatasaemia of infancy: Onica et al. reported on a 29-year-old man with immune deficiency. His bone alkaline phosphatase isoenzyme gave less precipitate with lectin than the bone isoenzyme of other patients with increased bone alkaline phosphatase but without evidence of a transient hyperphosphatasaemia (9).

The adult form of a transient hyperphosphatasaemia could be different from the condition in infants. The

\section{References}

1. Assanti R, Hultin H, Visakorpi JK. Serum alkaline phosphatase in healthy infants. Occurrence of abnormally high values without known cause. Ann Paediatr Fenn 1966; 12:139-42.

2. Stein P, Rosalki SB, Foo AY, Hjelm M. Transient hyperphosphatasemia of infancy and early childhood: clinical and biochemical features of 21 cases and literature review. Clin Chem 1987; 33:313-8.

3. Crofton PM. What is the cause of benign transient hyperphosphatasemia? A study of 35 cases. Clin Chem 1988; 34:33540.

4. Rosalki SB, Hurst NP. Transient presence in serum of an atypical alkaline phosphatase. Clin Chim Acta 1976; 73:149-55.

5. Chisholm JC. Transient, benign serum alkaline hyperphosphatasemia in an adult. J Natl Med Assoc 1986; 78:338-41.

6. Odeh MM, Bassan HM. Pleuropneumonia and alkaline phosphatase [letter]. Ann Intern Med 1988; 108:644.

7. Maekawa M, Sugiura K, Azuma Y, Kanno T. Benign transient hyperphosphatasemia in an adult with malignant lymphoma [letter]. Clin Chem 1989; 35:897.

8. Rosalki SB, Foo AY, Went J, Williams R, Baker DM. Transient hyperphosphatasemia of infancy and childhood in an adult [letter]. Clin Chem 1991; 37:1137-8. more widespread use of commercially available agarose gels, which allow affinity electrophoresis of alkaline phosphatase isoenzymes, will lead rapidly to the diagnosis of a transient hyperphosphatasaemia in infants, but may veil the true nature of this rare phenomenon in adulthood. The clinical laboratory has to be aware of a possibly atypical pattern.

\section{Acknowledgements}

We are indebted to Doctor Elisabeth Pohl (Transfusion Medicine, Surgical Department, University of Würzburg) for her kind cooperation and Doctor Viviane Van Hoof (Department of Clinical Chemistry, University Hospital Antwerp, Edegem, Belgium) for her useful correspondence.

9. Onica D, Torssander J, Waldenlind L. Recurrent transient hyperphosphatasemia of infancy in an adult. Clin Chem 1992; $38: 1913-5$.

10. Van Hoof VO, De Broe ME. Interpretation and clinical significance of alkaline phosphatase isoenzyme patterns. Crit Rev Clin Lab Sci 1994; 31:197-293.

11. Bergmeyer HU, Büttner H, Hillmann G, Kreutz FH, Lang $H$, Laue D, et al. Recommendations of the German Society for Clinical Chemistry. J Clin Chem Clin Biochem 1972; 10:281-91.

\section{Received January 3/April 2, 1997}

Corresponding author: Dr. Christian M. Schambeck, Zentrallabor der Medizinischen Universitätsklinik, Josef-Schneider-Strasse 2, D-97080 Würzburg, Germany

Fax: 0931-2012793, e-mail: c.schambeck@medizin.uniwuerzburg.de 